\title{
P21-activated protein kinase 1 induces colorectal cancer metastasis involving ERK activation and phosphorylation of FAK at Ser-910
}

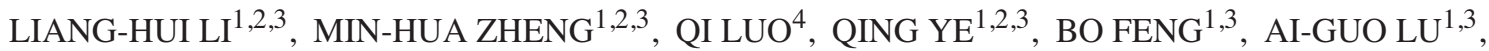 \\ MING-LIANG WANG ${ }^{1,3}$, XUE-HUA CHEN ${ }^{1,2}$, LI-PING SU ${ }^{1,2}$ and BING-YA LIU ${ }^{1,2}$ \\ ${ }^{1}$ Department of General Surgery, Ruijin Hospital, Shanghai Jiaotong University School of Medicine; ${ }^{2}$ Shanghai Institute \\ of Digestive Surgery; ${ }^{3}$ Shanghai Minimally Invasive Surgery Center, Shanghai 200025; ${ }^{4}$ Department of \\ General Surgery, Zhongshan Hospital, Xiamen University, Xiamen 361004, P.R. China
}

Received May 7, 2010; Accepted June 22, 2010

DOI: 10.3892/ijo_00000746

\begin{abstract}
Pak1 has been reported to be overexpressed in colorectal cancer, but the role of Pak1 in colorectal cancer remains unclear. In this study, Pak1 expression and activity were associated with aggressive behavior of colorectal cancer. Overexpression of Pak1 increased colorectal cancer cell motility and invasion, whereas down-regulation of Pak1 expression or activity reduced colorectal cancer cell migration and invasion. In addition, activated Pak1 inhibited stress fiber and focal adhesion complex formation in colorectal cancer cells and led to formation of motile phenotypes. Importantly, activated Pak1 elicited phosphorylation of FAK at Ser-910 via an ERK-dependent pathway in colorectal cancer cell lines and clinical samples. In conclusion, our results suggest that activated Pak1 regulates colorectal cancer metastasis requiring an ERK-dependent phosphorylation of FAK at Ser-910.
\end{abstract}

\section{Introduction}

As the third most common cancer worldwide, colorectal cancer is frequently complicated by metastatic disease (1). Despite unremitting efforts to improve a variety of diagnostic and therapeutic methods, the tumour metastasis after surgery is still the greatest threat to patients (2). Thus, elucidation of process and mechanism of CRC metastases is required for the ultimate control of CRC.

p21-activated kinase 1 (Pak1), the best characterized member of an evolutionary conserved family of serine/ threonine kinases (3), has been identified as an effector molecule for the small GTPases Rho, Rac1 and Cdc42 (4). Once

Correspondence to: Dr Min-Hua Zheng, Department of General Surgery, Ruijin Hospital, Shanghai Jiaotong University School of Medicine, Shanghai 200025, P.R. China

E-mail: eyelen@sina.com

Key words: colorectal cancer, metastasis, $\mathrm{P} 21$-activated protein kinase, ERK, FAK activated by one of these, Pak1 can mediate downstream signaling events involved in cytoskeletal reorganization, cell motility, apoptosis and transformation (5-7). Other signaling molecules can also affect Pak1 activity, including AKT, JAK2, PDK1 and PKA (8-11). Activation of Pak1 leads to autophosphorylation at several sites, including threonine-423 within the activation loop of the kinase (12).

Amplification of Pak1 has been found in breast cancer, kidney cancer, liver cancer and stomach cancer. Moreover, Pak1 has been reported to induce proliferation, motility and invasion of these cancer cells (13-16). Pak1 is believed to be involved in several cell signaling pathways, such as mitogenactivated protein kinases (MAPK) and nuclear factor- $\mathrm{kB}$ (NF-кB) $(3,17)$. Nevertheless, the exact molecular mechanism by which Pak1 contributes to carcinogenesis still remains ambiguous. More recently, Pak1 expression has been shown to significantly increase with progression of colorectal cancer to metastasis (18). The result suggests that Pak1 may play a key role in carcinogenesis and metastasis of colorectal cancer. Despite these reports to-date, studies focused on a possible role for Pak1 in human colorectal cancer are scarce. In this study, we found that Pak1 expression and activity were associated with aggressive behavior of colorectal cancer, and clinical follow-up showed that increased Pak-1 expression and activity were related to reduced patient survival. Ectopic expression of Pak1 obviously increased the motility and invasion of CRC cells, while down-regulation of Pak1 expression or activity impaired the motility and invasion of CRC cells. Moreover, Pak1 might affect metastasis of CRC cells by phosphorylation of FAK at Ser-910 via Erk signaling pathway. Our results may provide a therapeutic target for colorectal cancer and present a novel mechanism for Pak1 regulation of human colorectal cancer cell metastasis.

\section{Materials and methods}

Tissue samples. Surgical specimens of non-neoplastic and neoplastic colorectal tissues were obtained from the Xiamen University Zhongshan Hospital (Xiamen, China). Specimens were divided into two parts: one part was immediately frozen in liquid nitrogen and kept at $-70^{\circ} \mathrm{C}$, and the another was formalin-fixed and paraffin-embedded. The study protocol 
was approved by the Medical Ethics and Human Clinical Trial Committee at Xiamen University Zhongshan Hospital.

Cell cultures. SW1116, SW480 and LS174T human colon cancer cell lines were cultured in RPMI-1640 medium containing $10 \%$ fetal bovine serum (FBS). HT29 and HCT116 human colon cancer cell lines were maintained in DMEM medium supplemented with $10 \%$ FBS.

Materials. Anti-Pak1(N-20)(sc-882), anti-phospho-Pak1 (Thr423)(sc-12925), anti-DsRed (L-18) (sc-33353) and antiFAK (C-20) (sc-558) antibodies were obtained from Santa Cruz Biotechnology (Santa Cruz, CA). Anti-JNK, antiphospho-JNK, anti-p42/44MAPK, anti-phospho-p42/ 44MAPK, anti-p38MAPK and anti-phospho-p38MAPK antibodies were from Cell Signaling Technology (Beverly, MA). Anti-Rac1 and anti-Cdc42 antibodies were from BD Biosciences (Palo Alto, CA). Anti-phospho-FAK (Ser-910) antibody was from BioSource International (Camarillo, CA). Alexa Fluor ${ }^{\circledR 488}$ phalloidin was from Molecular Probes (Invitrogen Detection Technologies). Anti-paxillin antibody (Clone 5H11) was from Labvision (Neomarkers). Anti-mouse IgG FITC conjugate antibody was from Sigma (St. Louis, MO). Anti-glyceraldehyde-3-phosphate Dehydrogenase (GAPDH) antibody was from Kang Chen.

Plasmids. The Pak1 constructs, pDs-Red2-Pak1(WT) and pDs-Red2-Pak1(K299R) were generous gifts from Dr Jonathan Chernoff (Fox Chase Cancer Centre, Philadelphia, USA). Briefly, Pak1(WT) is a wild-type Pak and Pak1(K299R) lacks kinase activity $(1,19)$.

Transfection of cells. Cells were transfected with $4 \mu \mathrm{g}$ DNA constructs by using the Lipofectamine 2000 reagent, according to the manufacturer's instructions (Invitrogen, Carlsbad, CA). For constructing the stable transfectant, the transfected cells were selected in growth medium containing $1000 \mu \mathrm{g} / \mathrm{ml}$ Geneticin (G418; Life Technologies, Grand Island, NY). After 4-8 weeks, individual cell colonies were transferred for clone expansion and maintained in culture medium supplemented with $600 \mu \mathrm{g} / \mathrm{ml} \mathrm{G} 418$.

Pak1 siRNA. For RNA interference (RNAi) of Pak1, Pak1 small interfering RNA and control siRNA were purchased from Shanghai GenePharma Co., Ltd. Each of them (100 pmol in $2 \mathrm{ml}$ medium) was transfected by using Lipofectamine 2000 (Invitrogen).

Wound healing assay. Cells $\left(5 \times 10^{5}\right.$ per well) were seeded in 6-well plates and allowed to adhere for $24 \mathrm{~h}$. The cells were treated with $10 \mu \mathrm{g} / \mathrm{ml}$ Mitomycin C (Sigma) for $3 \mathrm{~h}$, washed with PBS, and then simply wounded with a pipette tip. Fresh, full medium was added, and the cells were allowed to close the wound for $48 \mathrm{~h}$. Photographs were taken every $24 \mathrm{~h}$ at the same position of the wound.

Cell motility and invasion assay. The polycarbonate membranes $6.5 \mathrm{~mm}$ in diameter with $8 \mu \mathrm{m}$ pores (Corning Costar, New York, NY, USA) coated with Matrigel (BD Biosciences, Bedford, MA) were used for invasion assay, whereas the membranes without Matrigel were for motility assay. After adding medium containing $10 \%$ FCS to the bottom chambers, single-cell suspensions in medium containing $0.1 \%$ BSA were seeded onto the filters $\left(1 \times 10^{5}\right.$ cells per each well $)$ and incubated for 24 or $48 \mathrm{~h}$ at $37^{\circ} \mathrm{C}, 5 \% \mathrm{CO}_{2}$. Filters were then washed and cells on the upper surface were removed with cotton swabs. The cells that had migrated or invaded to the lower surface of the filter inserts were fixed with $5 \%$ paraformaldehyde for $15 \mathrm{~min}$ and stained with $0.1 \%(\mathrm{w} / \mathrm{v})$ Crystal violet for $15 \mathrm{~min}$. The number of migrated or invaded cells was microscopically counted and 3 independent experiments were carried out to get an average cell number per high magnification field.

CCK-8 proliferation assay. The capability of cellular proliferation was measured by the CCK- 8 assay. Approximately $3 \times 10^{3}$ cells $/ 200 \mu 1$ were seeded into 96 -well culture plates at $0,24,48,72$ and $96 \mathrm{~h}$, respectively. Then cells were incubated with $20 \mu \mathrm{l}$ of the CCK- 8 solution at $37^{\circ} \mathrm{C}$ for $4 \mathrm{~h}$. The optical density (OD) was determined using a spectrophotometer (Bio-Rad) at a wavelength of $450 \mathrm{~nm}$. Finally, a calibration curve was prepared using the data obtained from the wells that contain known numbers of viable cells.

Colony-forming assay. The role of Pak1 on anchorageindependent growth was assayed using a soft agarose colonyforming assay. Briefly, an underlay of RPMI, 20\% FCS and $1.2 \%$ agarose was prepared in 6-well plates. Transfected cells were plated at a density of $4 \times 10^{3}$ cells/well in RPMI, $20 \%$ FCS and $0.6 \%$ agarose. Six-well plates were incubated in a humidified $\mathrm{CO}_{2}$ incubator at $37^{\circ} \mathrm{C}$ for 21 days, after which colonies were stained with $0.1 \%$ (w/v) Crystal violet for $15 \mathrm{~min}$ and counted.

Immunofluorescence assays and confocal microscopy. Cells were treated with lysophosphatidic acid (LPA) for $15 \mathrm{~min}$, fixed in $4 \%$ paraformaldehyde for $5 \mathrm{~min}$, and permeabilized with $0.1 \%$ Triton in PBS for 3 min. Alexa Fluor ${ }^{\circledR} 488$ phalloidin was used to visualize F-actin containing structures. Anti-paxillin combined with anti-mouse IgG FITC conjugate antibody were used to show focal adhesion complex. Immunofluorescence imaging was captured in a Zeiss LSM510 laser scanning confocal microscope (Carl Zeiss Microimaging Inc., Thornwood, NY).

Western blot analysis. Human tissue samples and cells were harvested into RIPA lysis buffer (Pierce) with freshly added protease inhibitor cocktail (Roche) and phosphatase inhibitor cocktail (Roche). The cell lysate was cleared by centrifugation at $4{ }^{\circ} \mathrm{C}$ and the supernatant was stored in small aliquots at $-80^{\circ} \mathrm{C}$. Normally, $20 \mu \mathrm{g}$ sample was loaded into each lane, separated by SDS-PAGE, transferred to polyvinylidene difluoride membrane, and probed with respective antibodies.

Immunohistochemistry. Sections were deparaffinized in xylene and hydrated through a graded series of alcohol to distilled water. Endogenous peroxidase was blocked by incubation in $0.3 \% \mathrm{H}_{2} \mathrm{O}_{2}$ in methanol for $30 \mathrm{~min}$. Antigen retrieval was performed by microwave irradiation in $0.01 \mathrm{M}$ citrate buffer, $\mathrm{pH}$ 6.0. Following washing 3 times with $1 \mathrm{X}$ PBS, non-specific 
binding was blocked with 5\% NGS in $1 \mathrm{X}$ TBS for $1 \mathrm{~h}$. To detect Pak1 expression, sections were incubated overnight at $4^{\circ} \mathrm{C}$ with anti-Pak1 specific polyclonal antibody (N-20 Santa Cruz) at a 1:50 dilution. Phosphorylated Pak1 was detected by a polyclonal anti-Pak1 phosphospecific antibody (sc12925 Santa Cruz) at a 1:150 dilution. Antibody binding was localized using a biotinylated secondary antibody, avidinconjugated HRP and DAB substrate. Slides were counterstained with hematoxylin and eosin. When viewed under light microscopy, positive cells stained brown. The semiquantitative evaluation of Stain was according to Carter et al (18).

Statistical analysis. Fisher's exact or $\chi^{2}$ test was used for statistical analysis of categorical data. The cancer-specific survival outcome was expressed by applying the KaplanMeier method, and the log-rank test was used to compare the prognostic significance of individual variables on survival. Statistical SPSS Version 15.0 was used to analyze data. A $\mathrm{p}<0.05$ was considered statistically significant.

\section{Results}

Pakl expression and activation correlated with clinicopathologic factors of human CRCs. To determine whether Pak1 was activated in colorectal cancer, immunohistochemical staining was done on 76 of paired samples of colorectal cancer and matched non-tumorous tissues, using anti-Pak1 antibody and anti-phospho-Pak1(Thr423) antibody, respectively. Positive staining was observed in the cytoplasm of colonic epithelial cells. Compared with matched non-tumorous tissues, $82.9 \%$ (63 of 76) of the CRC samples were found to have a higher expression of Pak1 (>2-fold) and $80.3 \%$ (61 of 76) of the CRC samples to have a higher expression of activated Pak1 (>2-fold). Overall, significantly stronger staining of Pak1 and activated Pak1 was observed in CRCs than in nontumorous tissues $(1.25 \pm 0.94$ vs. $0.16 \pm 0.39$ and $0.90 \pm 0.95$ vs. $0.09 \pm 0.28, \mathrm{p}<0.01$ and $\mathrm{p}<0.01$ Fig. 1). To further elucidate the correlation between expression level and activation of PAK1 and clinicopathologic factors in human CRC, 76 malignant tumors were further analyzed. The result showed that Pak1 expression and phosphorylation in tumor tissue were significantly associated with the depth of invasion $(\mathrm{p}=0.025$ and $\mathrm{p}=0.019)$, lymph node status $(\mathrm{p}<0.01$ and $\mathrm{p}=0.027)$, distant metastasis $(\mathrm{p}=0.028$ and $\mathrm{p}=0.016)$, tumor stages $(\mathrm{p}<0.01$ and $\mathrm{p}=0.013)$ and tumor grades $(\mathrm{p}=0.036$ and $\mathrm{p}<0.01)$. Nevertheless, Pak1 expression and phosphorylation were independent of tumor location, sex and age (Table I). Moreover, clinical follow-up was available for all but 3 cancer cases in this study and indicated that the 5-year survival rate of the lower-Pak1 expression and phosphorylation group were significantly better than that of the higher group $(58.5 \%$ vs. $34.4 \%$ and $67.4 \%$ vs. $14.8 \%, p=0.022$ and $p<0.01)$. The lower-Pak1 expression and phosphorylation group were defined as those at or below the median value (1.27 and 0.95 , respectively), whereas the higher group consisted of patients above the median value (Fig. 2).

Pakl enhanced cell migration and invasion. To elucidate the biological functions of Pak1 in colorectal cancer, we used the
A $\mathbf{N}$ T
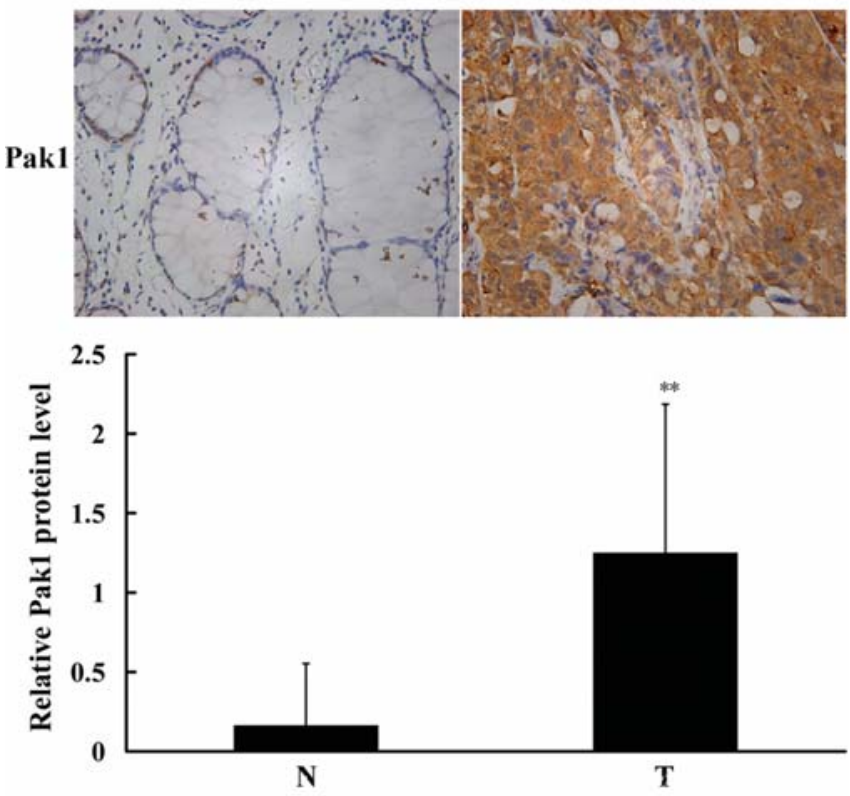

B N

$\mathbf{T}$
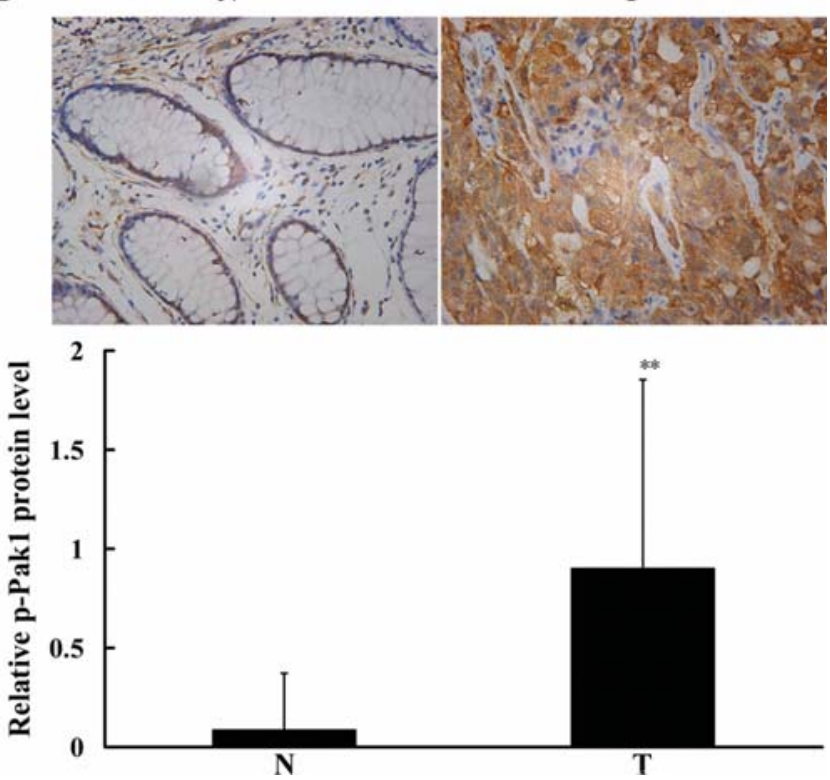

Figure 1. Imunohistochemical demonstration of Pak1 and phosphorylated Pak1 in human colorectal cancer. (A) Stronger staining of Pak1 is seen in cytoplasm of colorectal cancer, while very weak staining is observed in nontumorous tissue. Statistical analysis with relative expression level of Pak1 in 76 of paired samples of colorectal cancer and non-tumorous tissues. ${ }^{* *} \mathrm{P}<0.01$ (bottom). (B) Stronger staining of phosphorylated Pak1 is seen in cytoplasm of colorectal cancer, while very weak staining was observed in non-tumorous tissue. Statistical analysis with relative expression level of phosphorylated Pak1 in 76 of paired samples of colorectal cancer and nontumorous tissues. ${ }^{* *} \mathrm{P}<0.01$ (bottom). Original magnifications, $\mathrm{x} 200$ (top).

overexpression and antisense technique to specifically regulate Pak1 expression in CRC cells. We first detected Pak1 expression in 5 colorectal cancer cell lines (SW1116, SW480, HT29, LS174T and HCT116) by Western blotting. As shown in Fig. 3A, the expression of Pak1 was remarkably higher in HCT116 cells while was almost lost in SW1116 cells. Thus, SW1116 cells were transfected with plasmid DNA encoding 
Table I. Association of Pak1 expression and activation with clinicopathologic features in human CRCs.

\begin{tabular}{lcccc}
\hline Clinicopathological features & $\mathrm{n}$ & Pak1 & P-value & $\begin{array}{c}\text { p-Pak1 } \\
(\mathrm{x} \pm \mathrm{s})\end{array}$ \\
\hline
\end{tabular}

\section{Age}

$>60$ year

$\leq 60$ years

42

34

$1.49 \pm 0.89$

$1.50 \pm 0.93$

Sex

Male

Female

\section{3}

$1.22 \pm 0.91$

$1.35 \pm 0.09$

Depth of invasion

T1

T2

T3

T4

13

$0.60 \pm 0.60$

$1.21 \pm 0.81$

$1.32 \pm 1.11$

$1.37 \pm 0.98$

Lymph node status

N0
N1 2

Distant metastasis

$\begin{array}{lll}\text { M0 } & 58 & 1.06 \pm 0.89 \\ \text { M1 } & 18 & 1.87 \pm 0.80\end{array}$

Degrade

I

II

III

$\begin{array}{rr}9 & 0.50 \pm 0.31 \\ 56 & 1.35 \pm 0.94 \\ 811 & 1.35 \pm 1.05\end{array}$

Stage

A
B
C
D

$0.23 \pm 0.32$

$0.56 \pm 0.59$

$1.43 \pm 0.89$

$1.87 \pm 0.81$

Tumor location

$\begin{array}{lrr}\text { Rectum } & 18 & 1.15 \pm 0.93 \\ \text { Sigmoid colon } & 27 & 1.23 \pm 0.95 \\ \text { Descending colon } & 2 & 0.50 \pm 0.56 \\ \text { Transverse colon } & 12 & 1.30 \pm 0.86 \\ \text { Ascending colon } & 17 & 1.42 \pm 1.03\end{array}$

0.593

$0.95 \pm 1.01$

0.602

$0.98 \pm 0.86$

0.807

$0.96 \pm 0.92$

0.781

$0.97 \pm 1.04$

0.025

$0.43 \pm 0.68$

0.019

$1.09 \pm 0.88$

$1.20 \pm 1.37$

$1.24 \pm 1.03$

0.000

$0.60 \pm 0.78$

$1.12 \pm 1.02$

$1.22 \pm 0.92$

0.028

$0.64 \pm 0.88$

0.016

$1.34 \pm 1.02$

0.036

$0.73 \pm 0.72$

0.008

$0.84 \pm 0.87$

$1.75 \pm 1.06$

0.000

$0.33 \pm 0.49$

0.013

$0.49 \pm 0.69$

$1.10 \pm 0.94$

$1.34 \pm 1.02$

0.775

$0.74 \pm 0.89$

0.752

$1.09 \pm 0.94$

$0.95 \pm 1.20$

$1.02 \pm 0.95$

$0.95 \pm 1.04$
Pak1 full-length gene. The stable transfectant cell line SW1116-Pak1 was established and Pak1 expression was significantly up-regulated compared with SW1116 or the mock transfectant with pDsRed2 (named SW1116-DsRed2, Fig. 3A). Conversely, HCT116 cells were transfected with Pak1siRNA and specific knockdown of endogenous Pak1 in HCT116 cells was confirmed with Western blot analysis (Fig. 3D). To test whether Pak1 affects the motility of CRC cells, wound healing assay was performed with SW1116 cells, SW1116-DsRed2 cells and SW1116-Pak1 cells. In the presence of a cell division inhibitor, mitomycin C, SW1116Pak1 cells promoted wound healing significantly faster than
SW1116 cells and SW1116-DsRed2 cells $(355.62 \pm 14.15 \mu \mathrm{m}$ vs. $143.77 \pm 12.14 \mu \mathrm{m}$ and $98.68 \pm 12.20 \mu \mathrm{m}, \mathrm{p}<0.01$ and $\mathrm{p}<0.01$ Fig. 3B). Moreover, we found that up-regulation of Pak1 expression enhanced cell migration compared with either SW1116 or SW1116-DsRed2 (174.40 29.76 vs. $58.40 \pm 3.53$ and $39.40 \pm 8.10, p=0.037$ and $p=0.02$, Fig. $3 C)$, whereas knockdown of Pak1 significantly impaired the migratory capability compared with the control $(65.80 \pm 3.69$ vs. $158.40 \pm 8.65, \mathrm{p}<0.01$, Fig. $3 \mathrm{D})$. Then, the invasion assay was done and we observed that ectopic expression of Pak1 resulted in an increased invasion of SW1116-Pak1 cells compared with SW1116 cells or SW1116-DsRed2 cells 
A

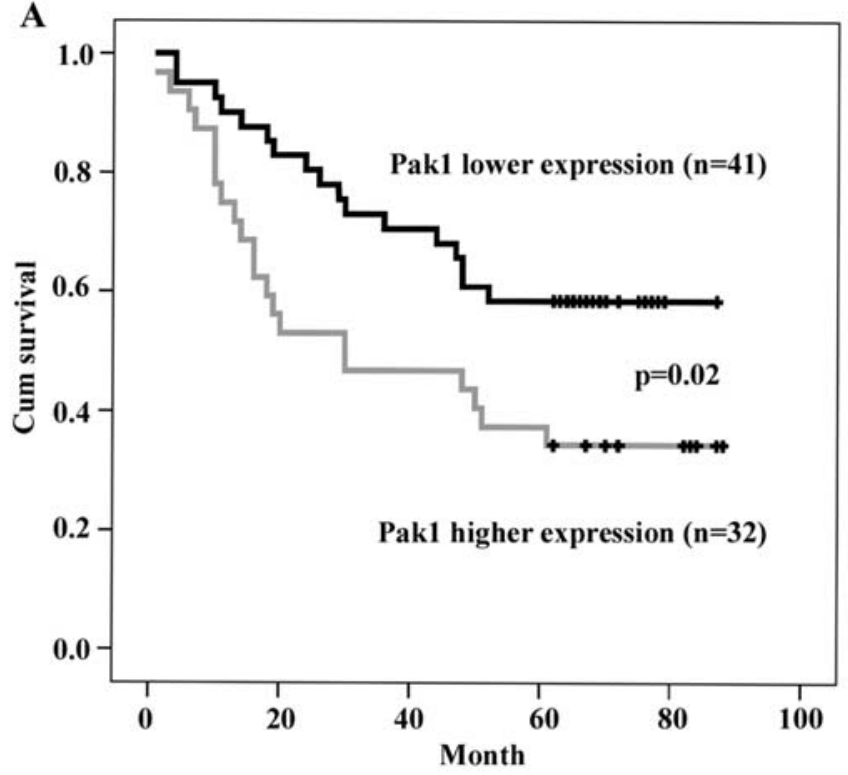

B

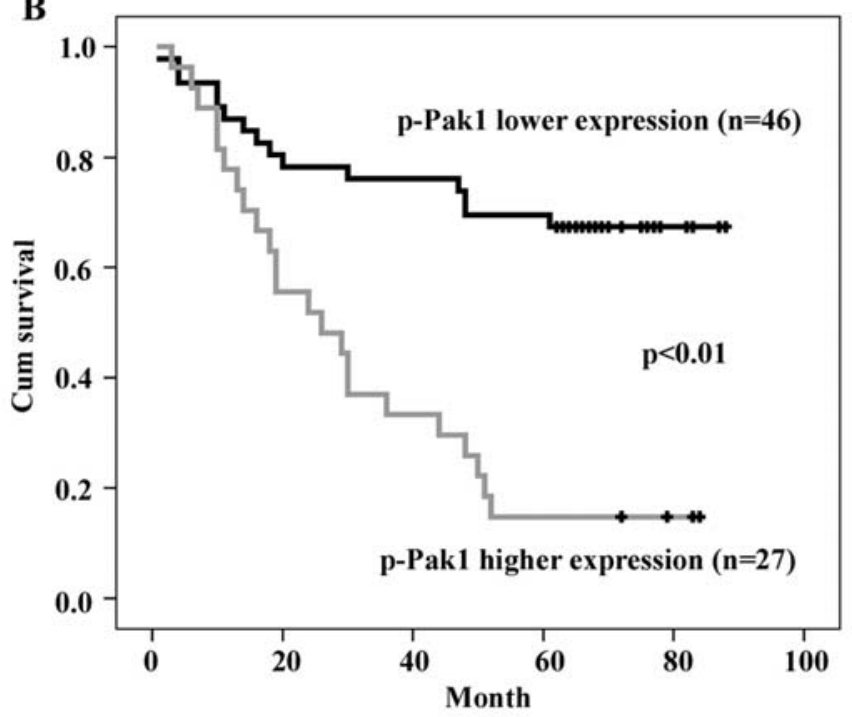

Figure 2. (A) Kaplan-Meier survival curves of Pak1 lower-expression group and Pak1 higher-expression group. (B) Kaplan-Meier survival curves of phosphorylated Pak1 lower-expression group and phosphorylated Pak1 higher-expression group.

$(138.20 \pm 4.44$ vs. $71.00 \pm 8.10$ and $66.40 \pm 6.39, p=0.001$ and p $<0.01$, Fig. 3E), whereas that of HCT116-Pak1siRNA cells was decreased when compared with the control $(55.80 \pm 3.90$ vs. $91.00 \pm 3.94, p<0.01$, Fig. 3F). To further examine if the Pak1 kinase activity was essential for the CRC cell migration and invasion, the Pak1 and a dominant-inactive form of Pak1 (Pak1K299R) were transiently transfected into SW1116 cells. As compared with the control, the Pak1 expression promoted the migration of SW1116 cells, whereas the Pak1K299R that lacks kinase activity inhibited their migration (101.20 \pm 9.05 vs.148.60 \pm 12.24 and $60.80 \pm 1.80, p=0.038$ and $p=0.022$ Fig. 3G). Similarly, Pak1 expression promoted the invasion of SW1116 cells, whereas Pak1 K299R inhibited their invasion $(92.80 \pm 9.03$ vs.138.20 \pm 4.44 and $62.40 \pm 5.59, \mathrm{p}<0.01$ and $\mathrm{p} \leq 0.01$, Fig. $3 \mathrm{H})$.
Activation of Pakl down-regulated stress fiber and focal adhesion complex formation. We tested if Pak1 regulated the reorganization of cytoskeleton components to increase the migration and invasion of CRC cells. As revealed by phalloidin staining, SW1116-Pak1 cells showed the presence of filopodia and much fewer stress fibers compared with SW1116-DsRed2 cells (Fig. 4A and B). As revealed by paxillin staining, the focal adhesion complex was fewer in the SW1116-Pak1 cells than SW1116-DsRed2 cells (Fig. 4C and D). Thus, we demonstrated that Pak1 effectively regulated reorganization of cytoskeleton components and stimulated motile phenotypes of CRC cells.

Pak1 had no effect on the proliferation rate of CRC cells. Overexpression of Pak1 has been reported to increase cell proliferation in breast cancer by up-regulating the expression of cyclin D1 (20) and in gastric cancer by up-regulating the expression of cyclin B1 (16). However, the role of Pak1 in the progression of CRC remained elusive. Therefore, we tested whether overexpression or downregulation of Pak1 could affect CRC growth. In CCK-8 assay, cells transfected with Pak1 full length cDNA or Pak1siRNA were found to grow similarly as the control cells, respectively (Fig. 5A and B). These results indicated that the expression level of Pak1 had no influence on cell growth. To further examine the effect of Pak1 on cell proliferation, colony-forming assay was perfomed. As shown in Fig. 5C, colony number of SW1116-Pak1 cells was similar as SW1116 cells or SW1116-DsRed2 cells $(37.00 \pm 2.41$ vs. $36.00 \pm 4.38$ and $36.00 \pm 3.22, p=0.978$ and $\mathrm{p}=0.967)$. In addition, neither cyclin B1 nor cyclin D1 was found significantly different in protein level between the control and SW1116-Pak1 cells (Fig. 5D).

Activation of ERK1/2 was required for Pakl-mediated migration and invasion of $C R C$. To identify the mechanism by which Pak1 could affect CRC cells migration and invasion, we investigated whether the activation of Pak1 regulated its well-defined downstream effectors, JNK, p38MAPK and extracellular signal regulated kinase (ERK). We observed that phosphorylation of Pak1 was obviously increased in Pak1 overexpressed cells compared with control cells, which suggested that the activity of Pak1 may provide an advantage for $\mathrm{CRC}$ cells to migrate. $\mathrm{Cdc} 42$ and Rac1, upstream regulators of Pak1, however, were not overexpressed in SW1116-Pak1 cells. ERK was remarkably phosphorylated in SW1116-Pak1 cells, whereas JNK or p38MAPK was not (Fig. 6A). To confirm the correlation between activation of Pak1 and phosphorylation of ERK in CRC, three pairs of human CRC clinical samples with Pak1 overexpression and one pair of CRC samples with normal expression of Pak1, were randomly selected for the analysis of phosphorylation of Pak1 and its downstream substrates and the same results were obtained (Fig. 6B). We next determined whether activation of ERK was required for Pak1-mediated migration and invasion of CRC, the migration and invasion of SW1116-Pak1 cells were specifically inhibited by ERK Inhibitor U0126, whereas p38 MAPK inhibitor SB203580 or JNK inhibitor SP600125 had very little effect on migration and invasion of SW1116-Pak1 cells (Fig. 6C and D). To further test if endogenous Pak1 activity regulates CRC cell migration through an ERKdependent pathway, HCT116 cells were treated with U0126, 


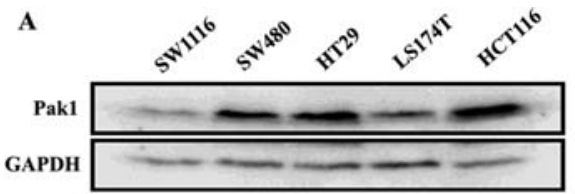

B

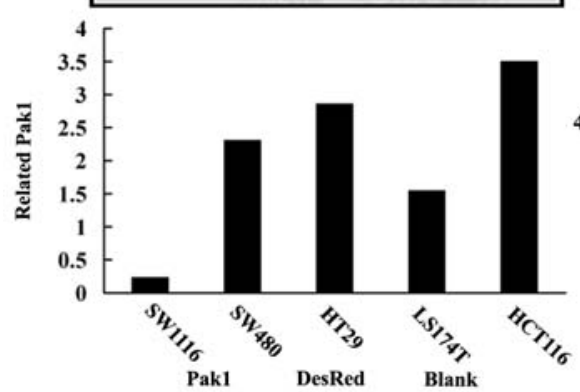

Oh
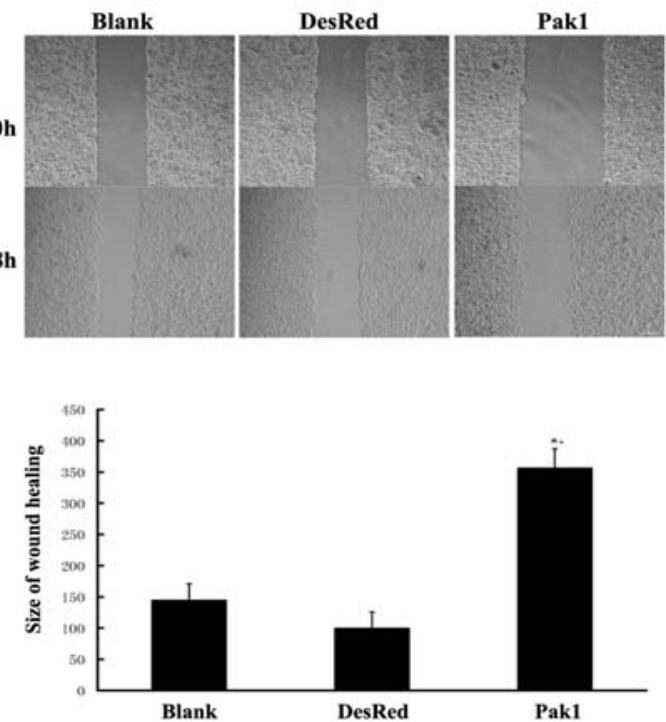

C

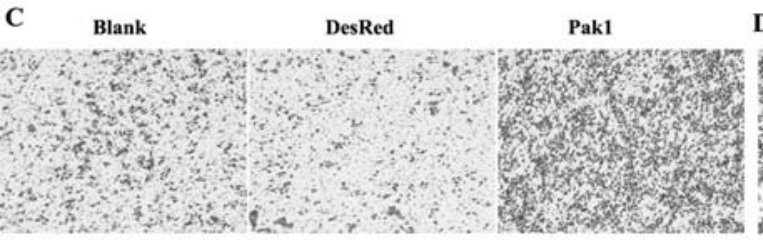

D Control siRNA
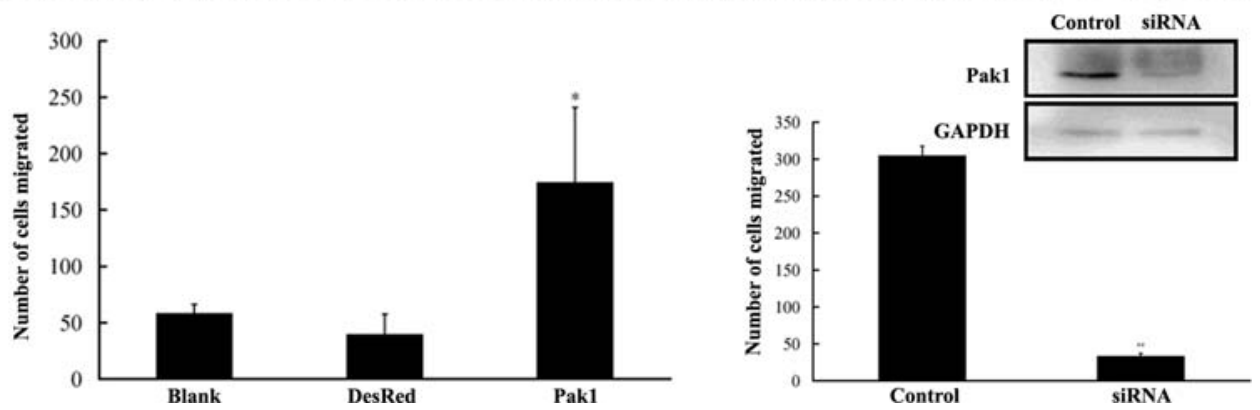

$\mathbf{E}$

E Blank

DesRed

Pak1

F Control

siRNA
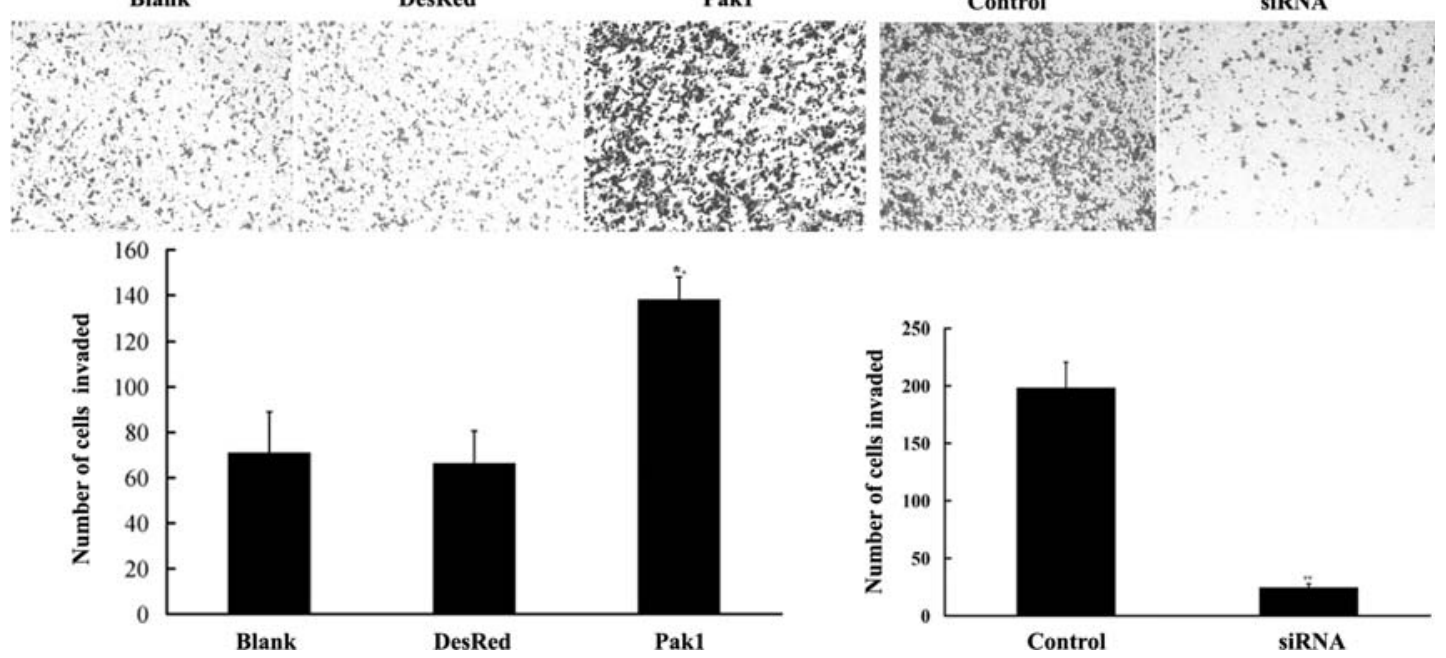

Figure 3. Pak1 induced CRC cells migration and invasion. (A) Western blot analysis demonstrates the protein level of Pak1 in the colorectal cancer cell lines (top). Relative protein level of Pak1 in CRC cell lines (insert). Expression of DsRed-Pak1 in SW1116 cells was confirmed by anti-DsRed antibody (bottom). (B) Wound healing assay. Representative images show size of the wound at time 0 and $48 \mathrm{~h}$ for SW1116, SW1116-DsRed2 and SW1116-Pak1 cells. Bar, 100 $\mu \mathrm{m}$ (top). Quantification of wound healing. ${ }^{* *} \mathrm{P}<0.01$ (bottom). (C) Representative images show SW1116, SW1116-DsRed2 and SW1116-Pak1 cells to migrate for $24 \mathrm{~h}$ (top). (D) Representative images show HCT116 cells transfected with Pak1siRNA and control siRNA to migrate for $24 \mathrm{~h}$ (top). Knockdown of Pak1 in HCT116 cells transfected with Pak1 siRNA was confirmed by Western blotting (inset). (E) Representative images show SW1116, SW1116DsRed2 and SW1116-Pak1 cells to invade for $48 \mathrm{~h}$ (top). (F) Representative images show HCT116 cells transfected with siRNA to invade for $48 \mathrm{~h}$ (top). 

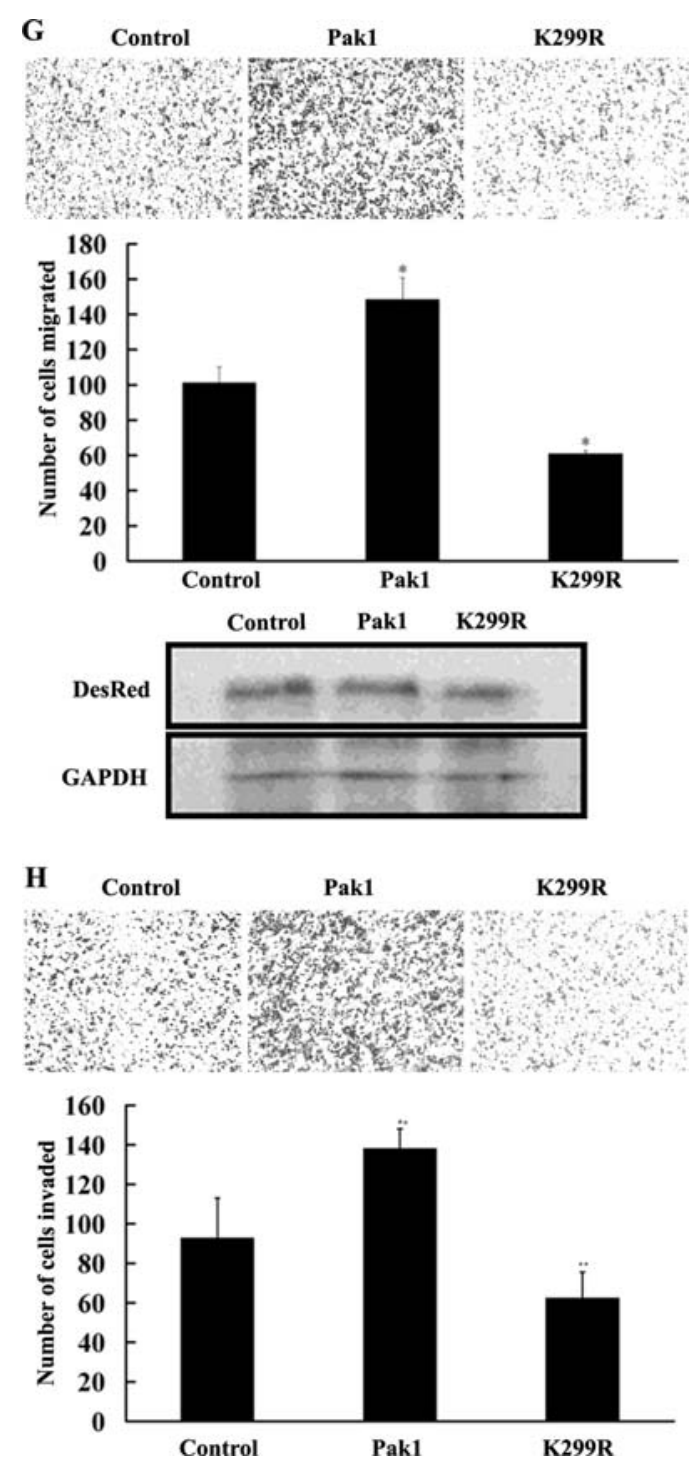

Figure 3. Continued. (G) Representative images show SW1116 cells transiently transfected with Pak1, Pak1K299R and empty vector to migrate for $24 \mathrm{~h}$ (top). Transient transfection of DsRed in SW1116 cells was confirmed by anti-DsRed antibody (insert). (H) Representative images show transiently transfected SW1116 cells to invade for $48 \mathrm{~h}$ (top). (C-H) Migrated or invaded cells of 6 randomly selected fields were counted. ${ }^{*} \mathrm{P}<0.05$, ${ }^{* *} \mathrm{P}<0.01$ (bottom).

SP600125 and SB203580, respectively. Similar to SW1116Pak1 cells, the migration and invasion of HCT116 cells were specifically inhibited by ERK inhibitor (Fig. 6E and F). Moreover, filopodia almost disappeared and focal adhesion complex was restored in SW1116-Pak1 cells treated with U0126 (Fig. 6G), suggesting that Pak1 regulates cytoskeletal reorganization through an ERK-dependent pathway which is involved in CRC cell migration and invasion.

Phosphorylation of FAK at Ser-910 by PAK1 via ERK pathway is important for CRC cell migration. It has been reported that reduced formation of focal adhesion contact is helpful for cell migration (20) and phosphorylation of FAK at Ser-910 via ERK inhibits the binding of paxillin to FAK and formation of focal adhesion complex (21). We therefore tested whether Pak1 regulates CRC migration via ERK phosphorylation of

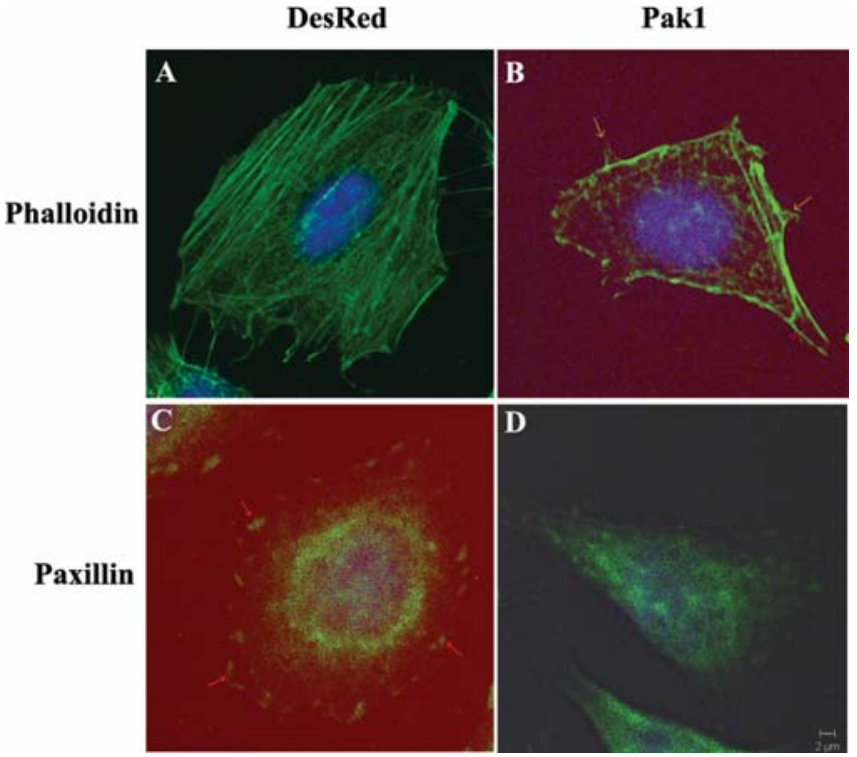

Figure 4. Pak1 regulation of cytoskeleton reorganization of SW1116 cells (A and B) The representative confocal images show the staining of F-actin containing structures. (C and D) The representative confocal images show the staining of focal adhesion complex. Bar, $2 \mu \mathrm{m}$.

FAK at Ser-910. Phosphorylation of FAK at Ser-910 was determined in SW1116, SW1116-Pak1 and SW1116-DsRed2 cells by Western blotting. As compared with the SW1116 and SW1116-DsRed2 cells, a striking increase in FAK Ser910 phosphorylation was detected in SW1116-Pak1 cells (Fig. 7A). To further determine whether Pak1 activity is required, phosphorylation of FAK at Ser-910 was detected in Pak1 and Pak1 K299R transfected SW1116 cells, respectively. Contrary to SW1116-Pak1 cells, SW1116-Pak1K299R cells showed markedly reduced phosphorylation of FAK at Ser910 (Fig. 7B), suggesting that the activation of Pak1 induces phosphorylation of FAK at Ser-910. To illustrate whether activation of Pak1 stimulates FAK phosphorylation at Ser-910 through an ERK-dependent pathway, SW1116-Pak1 cells were incubated for $1 \mathrm{~h}$ in the absence or presence of U0126 $(0.5-20 \mu \mathrm{M})$ and then phosphorylation of ERK and FAK at Ser-910 were analyzed by Western blotting. Phosphorylation of FAK at Ser-910 was observed to be sharply reduced in a concentration-dependent fashion due to similar reduction of phosphorylation of ERK. Almost complete inhibition of FAK phosphorylation at Ser-910 was achieved at $10 \mu \mathrm{M}$ (Fig. 7C). Furthermore, endogenous Pak1 was activated in HCT116 cells with epidermal growth factor (EGF) at $20 \mathrm{ng} / \mathrm{ml}$ for various times (0-40 min) and then lysates of these cells were subjected to Western blotting with respective antibodies. Our results showed that Pak1 phosphorylation was increased in a time-dependent manner and a maximal increase was achieved at $30 \mathrm{~min}$, and this was similar for the phosphorylation of ERK and FAK at Ser-910 (Fig. 7D), suggesting that activation of endogenous Pak1 induces the phosphorylation of ERK and FAK at Ser-910. Additionally, we used Pak1siRNA and ERK inhibitor U0126 (10 $\mu \mathrm{M}$, $30 \mathrm{~min}$ ) in HCT116 cells to specifically inhibit the activity of Pak1 and ERK, respectively. We found that inhibition of 

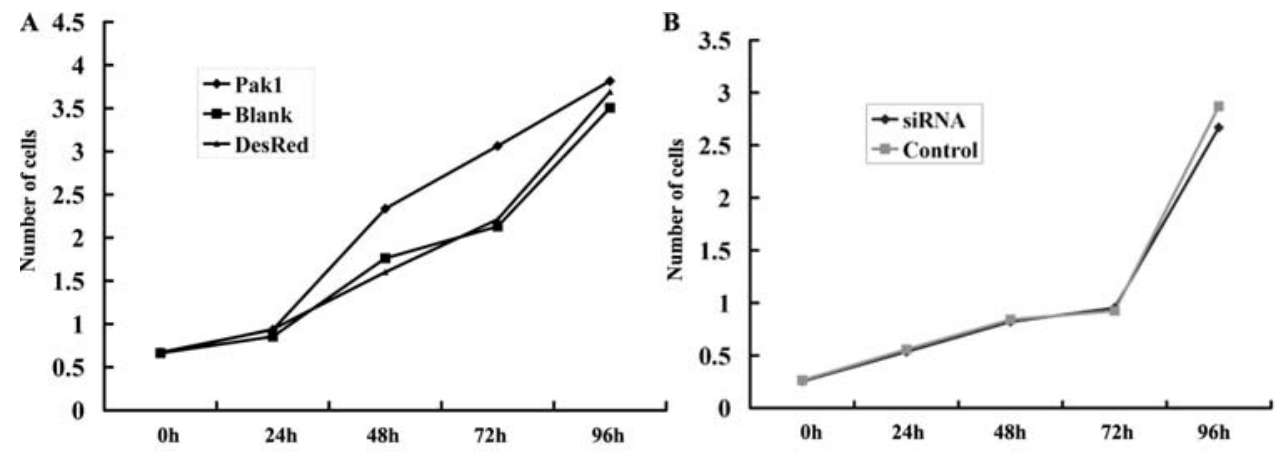

C

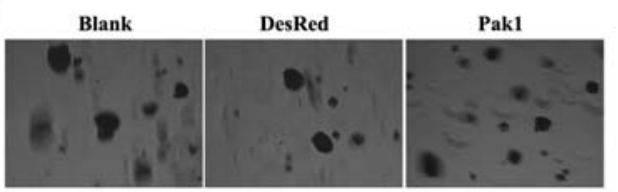

D
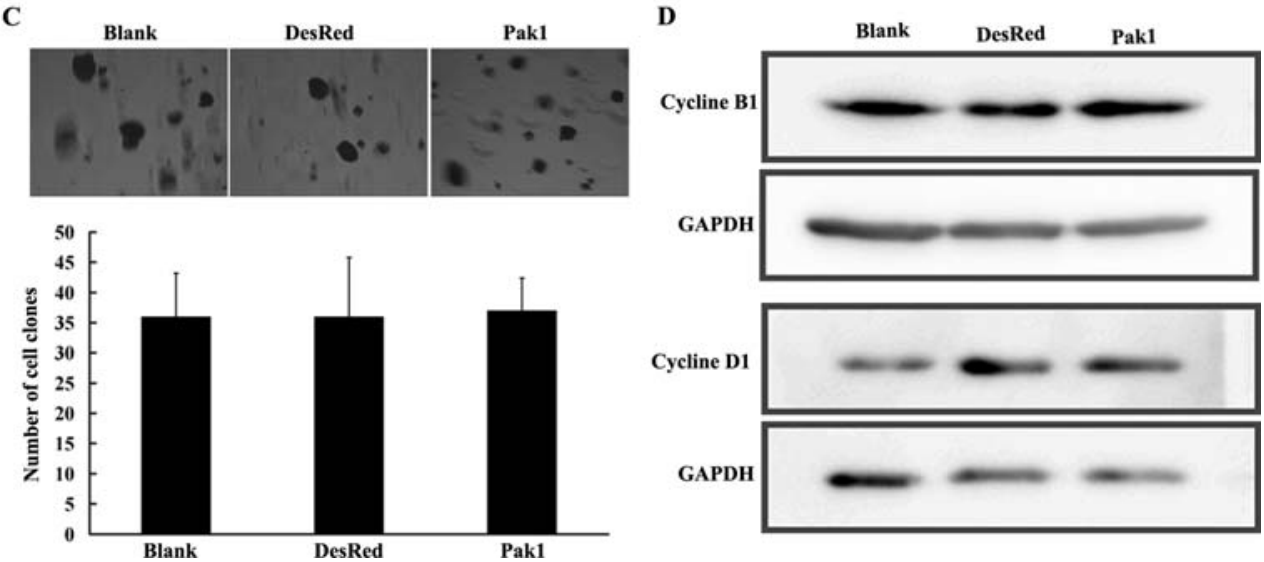

Figure 5. Effect of Pak1 on cell proliferation. (A) CCK-8 assay. SW1116, SW1116-DsRed2 and SW1116-Pak1 cells (3x10 $\left.{ }^{3}\right)$ were grown for $96 \mathrm{~h}$ and counted every 24 h. (B) CCK-8 assay. HCT116 cells transfected with Pak1 siRNA and control siRNA (3x103) were grown for $96 \mathrm{~h}$ and counted every $24 \mathrm{~h}$. (C) Anchorage-independent growth potential of SW1116, SW1116-DsRed2 and SW1116-Pak1 cells $\left(4 \times 10^{3}\right)$ was measured by their ability to form colonies on soft agar for 21 days (top). The number of colonies per plate is shown (bottom). Results shown are representative of three independent experiments. (D) The protein level of cycline B1 and cycline D1 was evaluated in SW1116-Pak1 cells and the control by Western blotting.
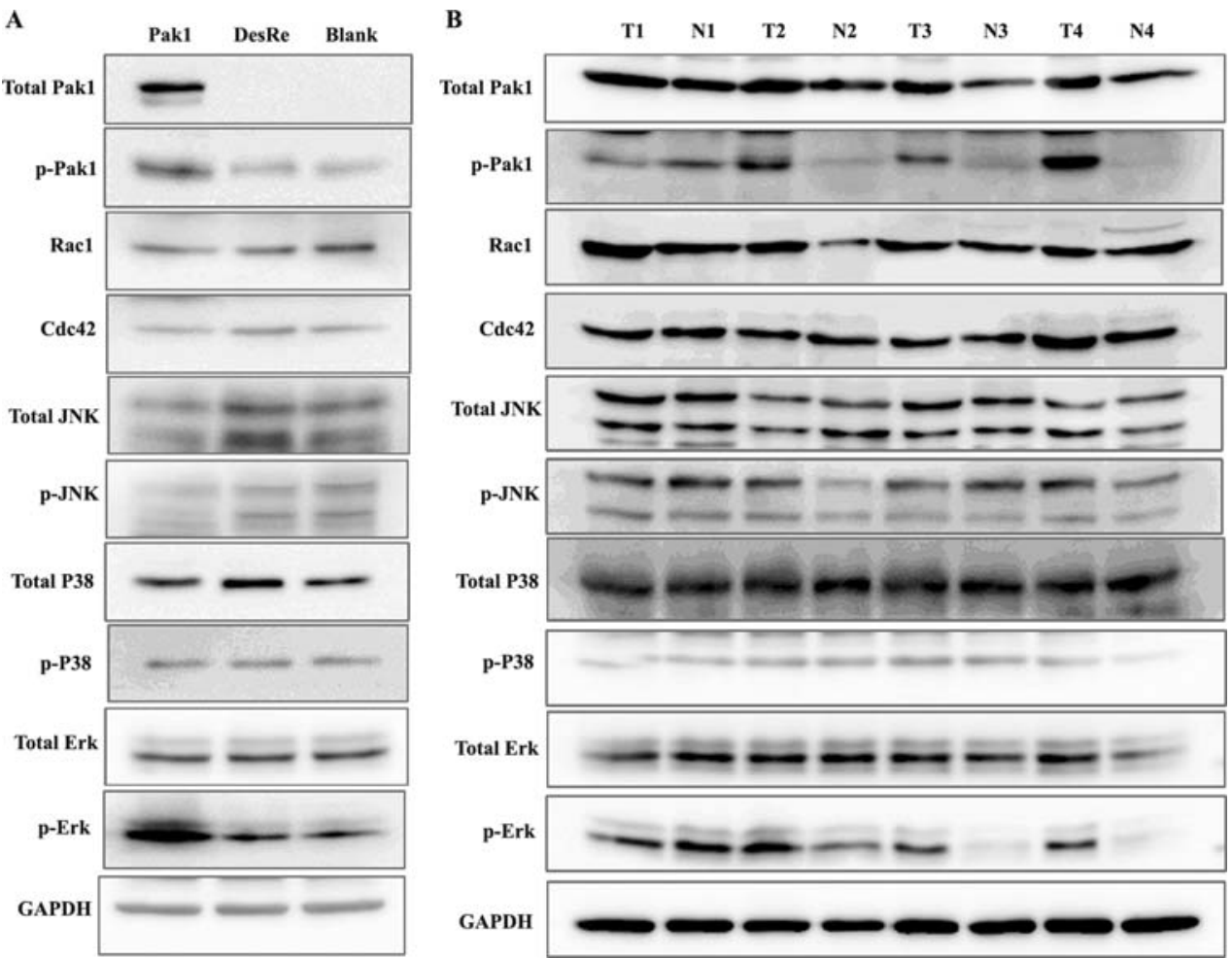

Figure 6. Pak1 regulated human CRC cell migration through an ERK-dependent pathway. (A) Western blotting analysis of Pak1, phospho-Pak1 (p-Pak1), Rac1, Cdc42, ERK1/2, phospho-ERK(p-ERK) 1/2, p38MAPK, phospho-p38MAPK, JNK, phospho-JNK (p-JNK) and GAPDH between SW1116, SW1116DsRed2 and SW1116-Pak1 cells. (B) Western blotting analysis of Pak1, p-Pak1, Rac1, Cdc42, ERK1/2, p-ERK, p38MAPK, p-p38MAPK, JNK, p-JNK and GAPDH between selected colorectal cancer and matched non-tumorous tissues. 

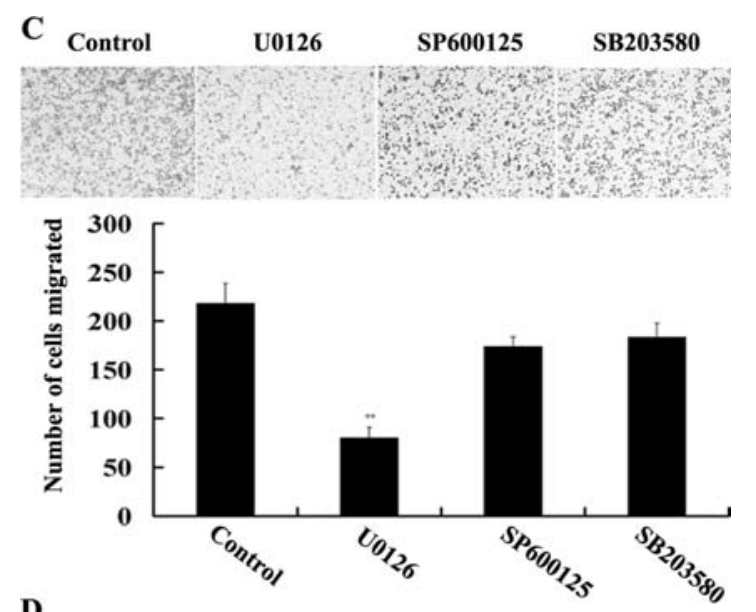

D
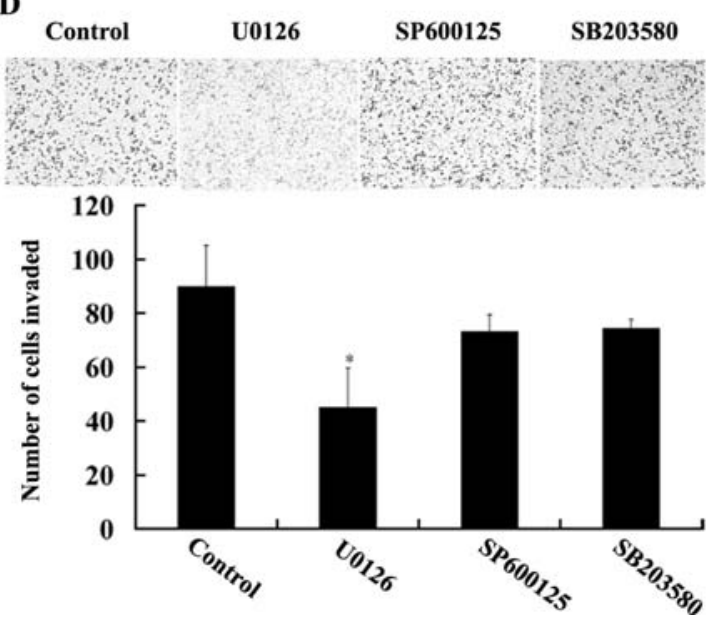

$\mathbf{G}$

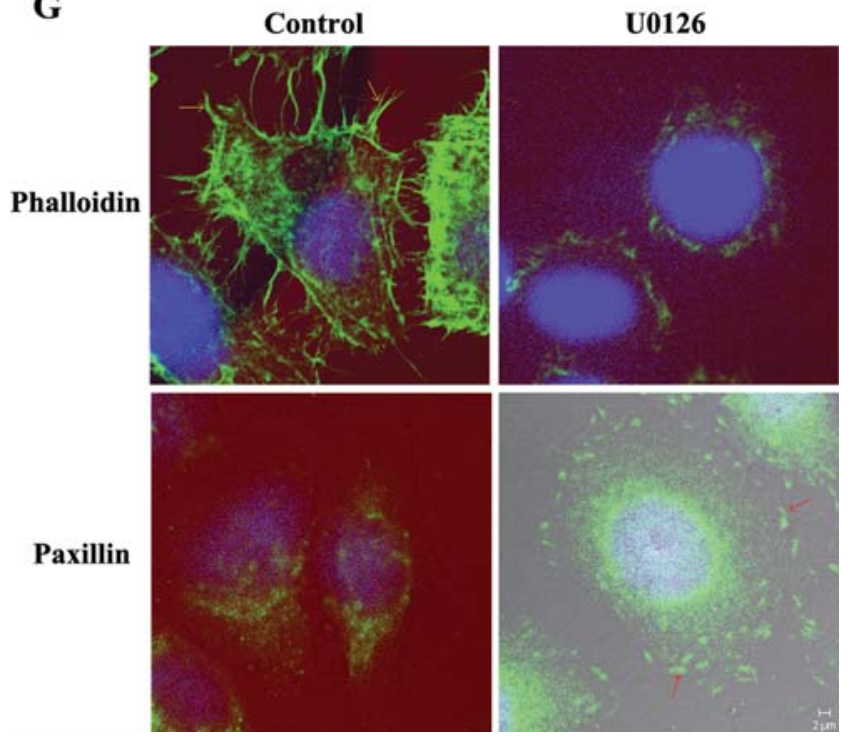

ERK activity almost completely abolished the phosphorylation of FAK at Ser-910, whereas knockdown of Pak1 expression substantially reduced activation of ERK and phosphorylation of FAK at Ser-910 (Fig. 7E). To further confirm the role of FAK phosphorylation at Ser-910 in CRC, we examined FAK Ser-910 in eight human CRCs (TNM stage I-IV each 2) by Western blotting. The results showed that the Ser-910-phosphorylated FAK was elevated with development of tumor stages (Fig. 7F). Taken together, these findings suggest a novel pathway in which Pak1 induces
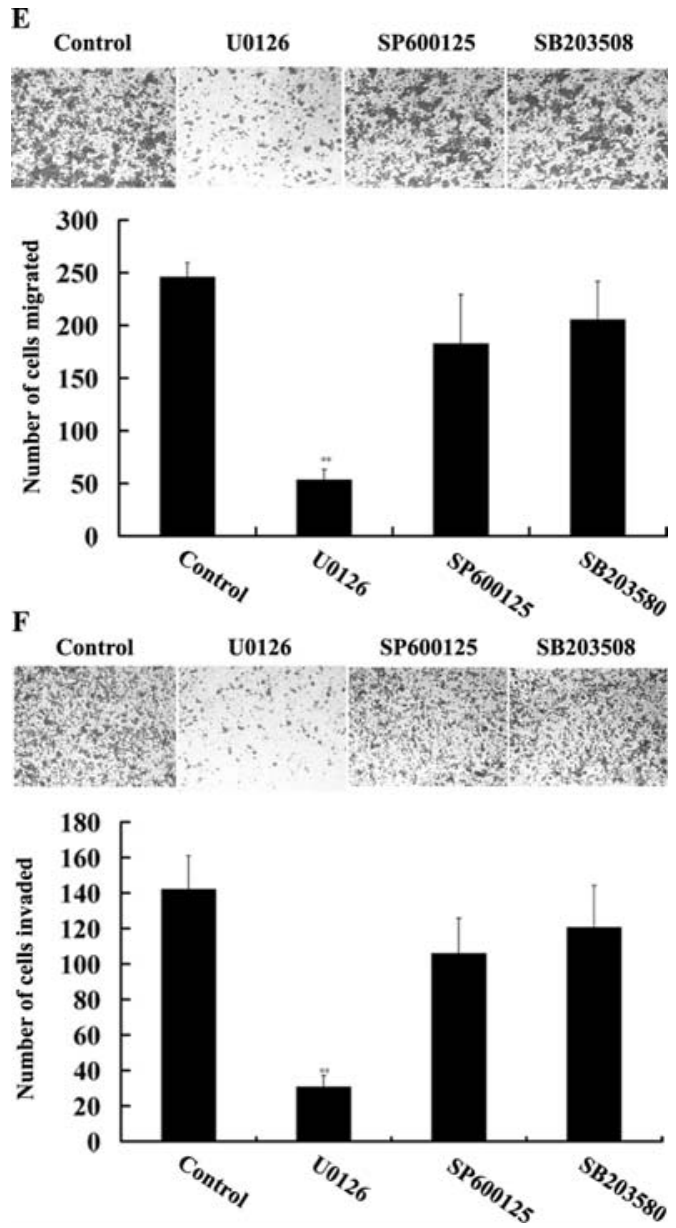

Figure 6. Continued. (C) SW1116-Pak1 cells were pretreated with pharmacologic inhibitors U0126 (10 $\mu \mathrm{mol} / 1$; ERK inhibitor), SB203580 $(2.5 \mu \mathrm{mol} / 1$; p38 MAPK inhibitor) and SP600125 (50 $\mu \mathrm{mol} / 1$; JNK inhibitor), respectively, and then were allowed to migrate for $24 \mathrm{~h}$ (top). (D) SW1116-Pak1 cells were pretreated with pharmacologic inhibitors as $\mathrm{C}$, and then were allowed to invade for $48 \mathrm{~h}$ (top). (E) HCT116 cells were pretreated with pharmacologic inhibitors as $\mathrm{C}$, and then were allowed to migrate for $24 \mathrm{~h}$ (top). (F) HCT116 cells were pretreated with pharmacologic inhibitors as C, and then were allowed to invade for $48 \mathrm{~h}$ (top). (C-F) Quantification of three independent experiments. ${ }^{*} \mathrm{P}<0.05,{ }^{* *} \mathrm{P}<0.01$, statistical significance between the DMSO control and U0126 (bottom). (G) SW1116-Pak1 cells were pretreated with U0126 and then were stained with Alexa Fluor ${ }^{\circledR} 488$ phalloidin to visualize F-actin containing structures and with anti-paxillin antibody to visualize focal adhesion complex, DMSO as a control. Bar, $2 \mu \mathrm{m}$.

activation of ERK, which in turn phosphorylates FAK at Ser910, thus promoting CRC cell migration.

\section{Discussion}

Although Pak1 overexpression has been reported to be correlated with progression of colorectal cancer, virtually nothing is known about the role of Pak1 in colorectal cancer. In this study, we found that Pak1 expression was significantly increased in CRCs, and closely correlated with aggressive 
A

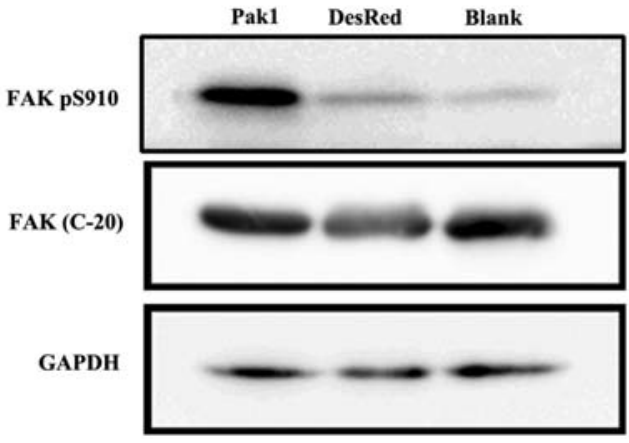

C
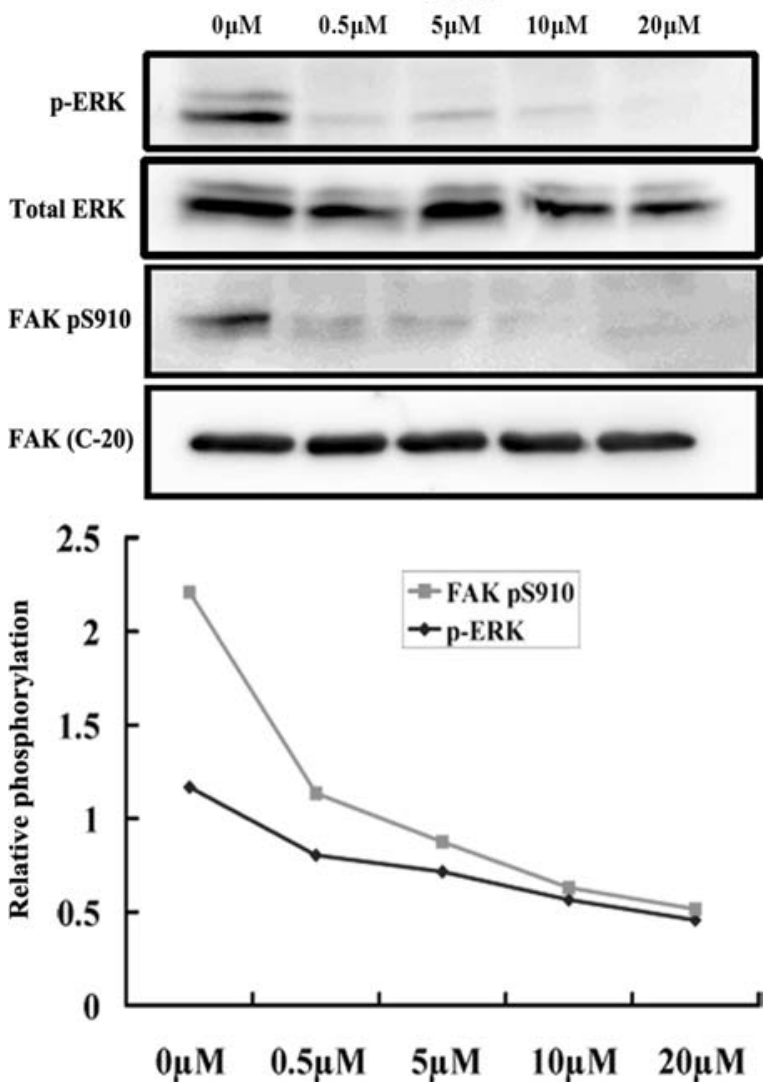

B

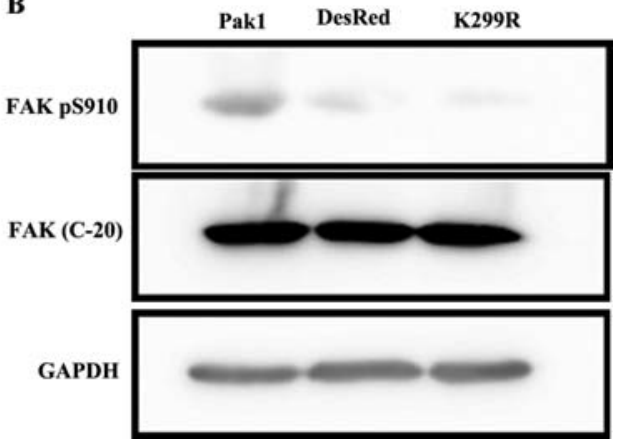

D

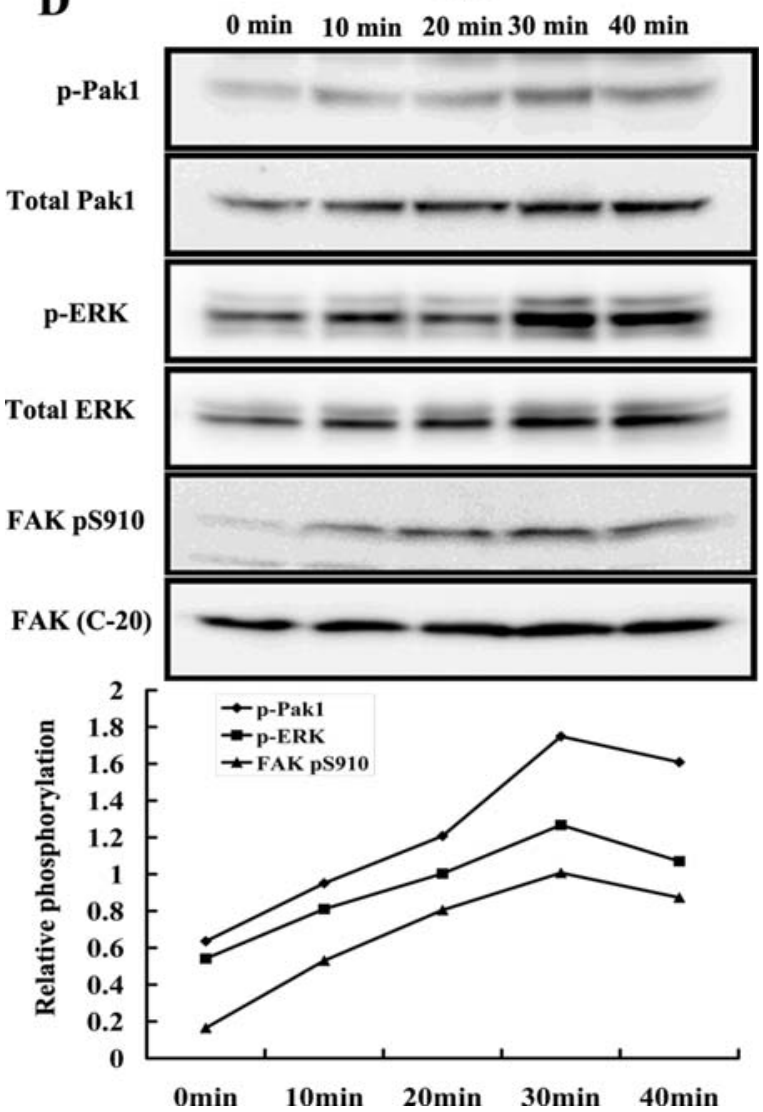

Figure 7. Pak1-mediated CRC migration required phosphorylation of FAK at Ser-910. (A) Western blot analysis of FAK phosphorylation at Ser-910 and FAK in SW1116, SW1116-DsRed2 and SW1116-Pak1 cells. (B) Western blotting analysis of FAK phosphorylation at Ser-910 and FAK in SW1116 cells transiently transfected with Pak1 and Pak1K299R. (C) HCT116 cells were treated with increasing concentrations of U0126 at $37^{\circ} \mathrm{C}$ for $1 \mathrm{~h}$ and were subsequently lysed. Western blot analysis of p-ERK, ERK, p-FAK Ser-910 and FAK (top). Quantification of inhibition of p-FAK Ser-910 and p-ERK by U0126 in a concentration-dependent manner (bottom). (D) HCT116 cells were serum starved and treated with EGF (20 ng/ml) for various times as indicated and were subsequently lysed. Western blot analysis of p-Pak1, Pak1, p-ERK, ERK, p-FAK Ser-910 and FAK (top). Quantification of p-Pak1, p-ERK and p-FAK Ser-910 induced by EGF at various times (bottom).

progression of colorectal cancer. Interestingly, Pak1 activity was also significantly associated with depth of invasion, lymph node status, distant metastasis, tumor stages, tumor grades and 5-year survival rate. Moreover, CRC cells with Pak1 overexpression had higher migration rate, whereas those with down-regulation of Pak1 expression and activation had significantly reduced cell migration rate. Previous documents have reported that cytoskeleton reorganization is a fundamental component of several cellular processes, including cell motility $(22,23)$. A kinase-dead Pak1 mutant was transfected into highly invasive breast cancer cells and then resulted in the suppression of motile phenotypes as well as invasiveness of cells by regulating cytoskeleton reorganization (24), whereas kinase-active PAK1 led to opposite results in breast cancer cells $(7,25)$. In our study, Pak1 effectively induced formation of filopodia and disassembly of stress fiber and focal adhesion complex in SW1116 cells, thereby resulting in motile phenotypes of cells. Pak1 has been reported to regulate several signaling pathways in carcinogenesis. Pak1 stimulates the proliferation of renal cell carcinoma in a preferential ERK-sensitive manner (14). Pak1 modulating hepatocellular carcinoma metastasis may be associated with activation of JNK and phosphorylation of paxillin (15). In this study, we observed that ERK was activated 

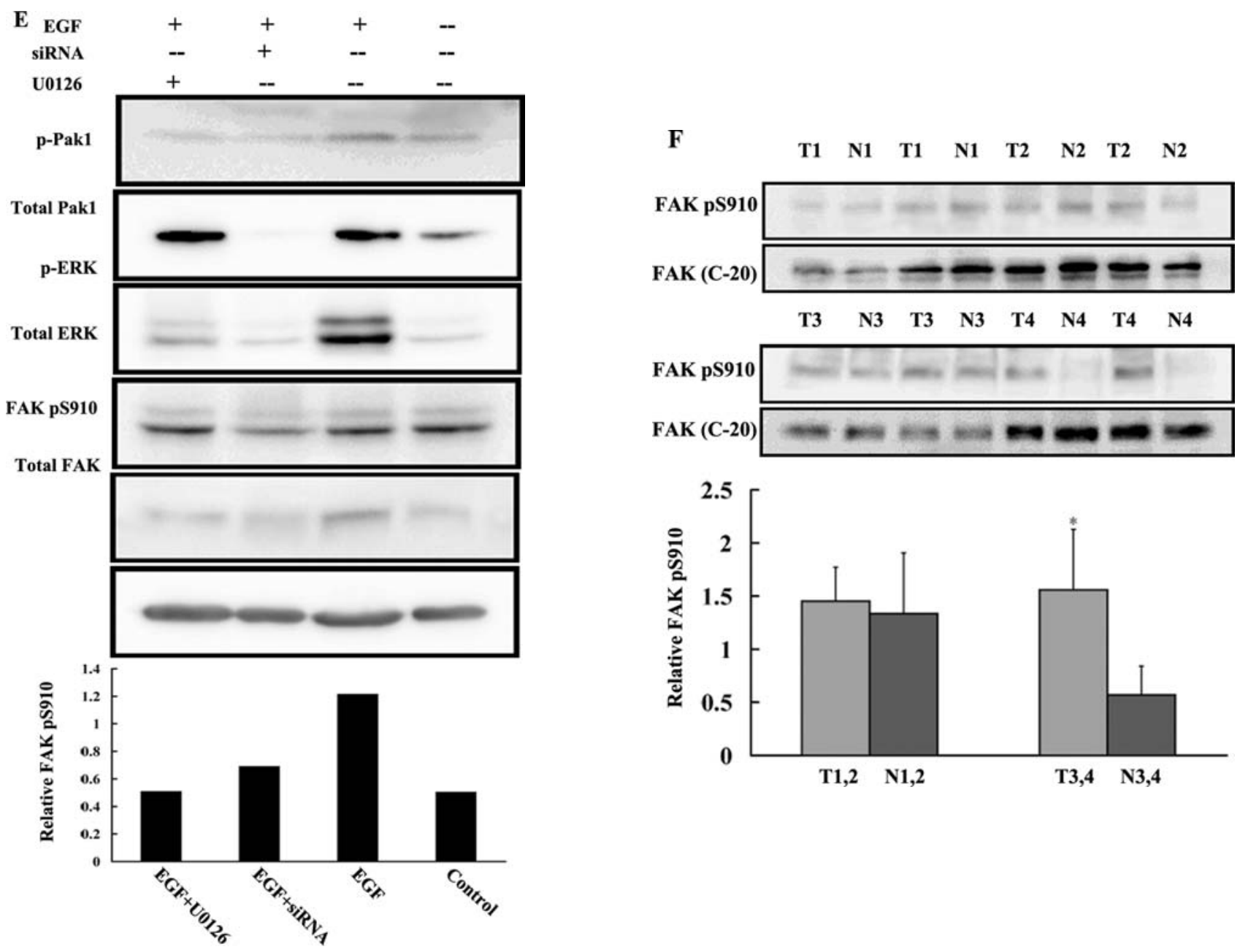

$\begin{array}{llllllll}\text { T3 } & \text { N3 } & \text { T3 } & \text { N3 } & \text { T4 } & \text { N4 } & \text { T4 } & \text { N4 }\end{array}$
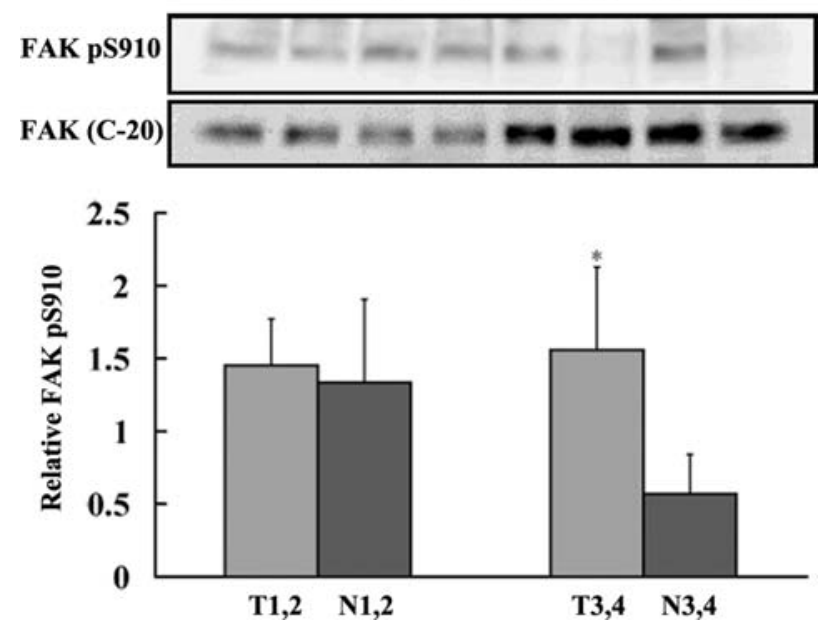

Figure 7. Continued. (E) HCT116 cells were serum starved and treated with EGF $(20 \mathrm{ng} / \mathrm{ml}$; for 30 min). Alternatively, HCT116 cells were pretreated with U0126 for $2 \mathrm{~h}$ followed by treatment of EGF or transfected with Pak1siRNA. Western blotting was done using indicated antibodies (top). Quantification of relative FAK phosphorylation at Ser-910 (bottom). (F) Western blot analysis of p-FAK Ser-910 and FAK in colorectal cancer and matched non-tumorous tissues. T1-T4, Dukes stage I-IV colorectal cancer tissue; N1-N4, matched non-tumorous tissues (top). Quantification of relative FAK phosphorylation at Ser-910 (bottom).

in both clinical CRC samples and CRC cells overexpressing Pak1, whereas JNK or p38MAPK was not.

Furthermore, ERK-dependent pathway was required in Pak1-mediated CRC cells migration and cytoskeletal reorganization. Recent studies showed that EGF induces FAK phosphorylation at Ser-910 through an ERK-dependent pathway, inhibiting the ability of FAK to associate with paxillin and formation of focal adhesion complex $(21,26)$. The reduced formation of focal adhesion contact is helpful for cell migration (20). Given that Pak1 may be activated by EGF (27), it is reasonable that the activated Pak1 is involved in phosphorylation of FAK at Ser-910 via activation of ERK, thereby promoting CRC cell migration. We offered the evidence, for the first time, that activation of Pak1 induced phosphorylation of FAK at Ser-910 through an ERK-dependent pathway in CRC cells. Our findings also showed that the phosphorylation level of FAK at Ser-910 was higher in Pak1 overexpressing CRC cells with the highly metastatic property, but was attenuated in Pak1K299R transfected CRC cells with poorly metastatic potential. In CRC samples, the Ser-910-phosphorylated FAK was also elevated with development of tumor stages. Ser-843, the other serine residue of FAK, has also been reported to be phosphorylated in epithelial cells through a $\mathrm{Ca}^{2+}$-dependent manner (26). The elucidation of the precise role of FAK phosphorylation at Ser-843 in epithelial cells warrants further study. Although Pak1 has been identified as the downstream effector targets of Rac1 and Cdc42 in mammalian cells (22), no significant difference of these regulators at protein level was observed in our present study. Given that Pak1 is also activated by several other physiological signals, such as guanine nucleotide factor pix (28), filamin A (29), adaptor protein Nck (30) and phosphatidylinositol 3-kinase/Akt (10), activation of Pak1 in CRC cells may be induced through Cdc42/Rac1-independent mechanisms. Although Pak1 has been reported to be involved in cell proliferation $(7,14,16)$, we observed little difference in the cell proliferation rates between Pak1 stable cells and control cells, suggesting that Pak1 may play different roles in the development of CRC. In conclusion, we have shown that Pak1 plays an important role in cancer metastasis. The mechanism by which Pak1 induces cancer metastasis may involve activation of ERK and phosphorylation of FAK at 
Ser-910. Therefore, Pak1 may be a useful prognostic marker and therapeutic target of human CRC.

\section{Acknowledgements}

We thank Dr Chao Pan (Department of Pathology, Zhongshan Hospital, Xiamen University) for assistance in acquisition and evaluation of the archival tumor specimens used in this study. We also thank Dr Jun Ji, Dr Bei Q Yu and Dr Lei Guo (Institute of Digestive Surgery, Ruijin Hospital) for excellent technical assistance and advice.

\section{References}

1. Abdalla EK, Adam R, Bilchik AJ, Jaeck D, Vauthey JN and Mahvi D: Improving resectability of hepatic colorectal metastases: expert consensus statement. Ann Surg Oncol 13: 1271-1280, 2006.

2. Gennari L, Russo A and Rossetti C: Colorectal cancer: what has changed in diagnosis and treatment over the last 50 years? Tumori 93: 235-241, 2007.

3. Kumar R, Gururaj AE and Barnes CJ: p21-activated kinases in cancer. Nat Rev Cancer 6: 459-471, 2006.

4. Narumiya S, Ishizaki $\mathrm{T}$ and Watanabe N: Rho effectors and reorganization of actin cytoskeleton. FEBS Lett 410: 68-72, 1997.

5. Kumar R and Vadlamudi RK: Emerging functions of p21activated kinases in human cancer cells. J Cell Physiol 193: 133-144, 2002.

6. Schurmann A, Mooney AF, Sanders LC, et al: p21-activated kinase 1 phosphorylates the death agonist bad and protects cells from apoptosis. Mol Cell Biol 20: 453-461, 2000.

7. Vadlamudi RK, Adam L, Wang RA, et al: Regulatable expression of $\mathrm{p} 21$-activated kinase-1 promotes anchorageindependent growth and abnormal organization of mitotic spindles in human epithelial breast cancer cells. J Biol Chem 275: 36238-36244, 2000.

8. Zhou GL, Zhuo Y, King CC, Fryer BH, Bokoch GM and Field J: Akt phosphorylation of serine 21 on Pak1 modulates Nck binding and cell migration. Mol Cell Biol 23: 8058-8069, 2003.

9. Rider L, Shatrova A, Feener EP, Webb L and Diakonova M: JAK2 tyrosine kinase phosphorylates PAK1 and regulates PAK1 activity and functions. J Biol Chem 282: 30985-30996, 2007.

10. King CC, Gardiner EM, Zenke FT, et al: p21-activated kinase (PAK1) is phosphorylated and activated by 3-phosphoinositidedependent kinase-1 (PDK1). J Biol Chem 275: 41201-41209, 2000.

11. Howe AK and Juliano RL: Regulation of anchorage-dependent signal transduction by protein kinase A and p21-activated kinase. Nat Cell Biol 2: 593-600, 2000.

12. Chong C, Tan L, Lim L and Manser E: The mechanism of PAK activation. Autophosphorylation events in both regulatory and kinase domains control activity. J Biol Chem 276: 17347-17353, 2001.

13. Bekri S, Adelaide J, Merscher S, et al: Detailed map of a region commonly amplified at 11q13->q14 in human breast carcinoma. Cytogenet Cell Genet 79: 125-131, 1997.
14. O'Sullivan GC, Tangney M, Casey G, Ambrose M, Houston A and Barry OP: Modulation of p21-activated kinase 1 alters the behavior of renal cell carcinoma. Int J Cancer 121: 1930-1940, 2007.

15. Ching YP, Leong VY, Lee MF, Xu HT, Jin DY and Ng IO: $\mathrm{P} 21$-activated protein kinase is overexpressed in hepatocellular carcinoma and enhances cancer metastasis involving c-Jun NH2-terminal kinase activation and paxillin phosphorylation. Cancer Res 67: 3601-3608, 2007.

16. Liu F, Li X, Wang C, et al: Downregulation of p21-activated kinase-1 inhibits the growth of gastric cancer cells involving cyclin B1. Int J Cancer 125: 2511-2519, 2009.

17. Frost JA, Xu S, Hutchison MR, Marcus S and Cobb MH: Actions of Rho family small $G$ proteins and p21-activated protein kinases on mitogen-activated protein kinase family members. Mol Cell Biol 16: 3707-3713, 1996.

18. Carter JH, Douglass LE, Deddens JA, et al: Pak-1 expression increases with progression of colorectal carcinomas to metastasis. Clin Cancer Res 10: 3448-3456, 2004.

19. Sells MA, Knaus UG, Bagrodia S, Ambrose DM, Bokoch GM and Chernoff J: Human p21-activated kinase (Pak1) regulates actin organization in mammalian cells. Curr Biol 7: 202-210, 1997.

20. Ilic D, Furuta Y, Kanazawa S, et al: Reduced cell motility and enhanced focal adhesion contact formation in cells from FAKdeficient mice. Nature 377: 539-544, 1995.

21. Hunger-Glaser I, Salazar EP, Sinnett-Smith J and Rozengurt E: Bombesin, lysophosphatidic acid, and epidermal growth factor rapidly stimulate focal adhesion kinase phosphorylation at Ser910: requirement for ERK activation. J Biol Chem 278: 22631-22643, 2003.

22. Hall A: Rho GTPases and the actin cytoskeleton. Science 279: 509-514, 1998.

23. Sells MA, Boyd JT and Chernoff J: p21-activated kinase 1 (Pak1) regulates cell motility in mammalian fibroblasts. J Cell Biol 145: 837-849, 1999.

24. Adam L, Vadlamudi R, Mandal M, Chernoff J and Kumar R: Regulation of microfilament reorganization and invasiveness of breast cancer cells by kinase dead p21-activated kinase-1. J Biol Chem 275: 12041-12050, 2000.

25. Adam L, Vadlamudi R, Kondapaka SB, Chernoff J, Mendelsohn $\mathrm{J}$ and Kumar R: Heregulin regulates cytoskeletal reorganization and cell migration through the p21-activated kinase-1 via phosphatidylinositol-3 kinase. J Biol Chem 273: 28238-28246, 1998.

26. Jiang X, Sinnett-Smith J and Rozengurt E: Differential FAK phosphorylation at Ser-910, Ser-843 and Tyr-397 induced by angiotensin II, LPA and EGF in intestinal epithelial cells. Cell Signal 19: 1000-1010, 2007.

27. He H, Levitzki A, Zhu HJ, Walker F, Burgess A and Maruta H: Platelet-derived growth factor requires epidermal growth factor receptor to activate p21-activated kinase family kinases. J Biol Chem 276: 26741-26744, 2001.

28. Bagrodia $S$ and Cerione RA: Pak to the future. Trends Cell Biol 9: 350-355, 1999.

29. Vadlamudi RK, Li F, Adam L, et al: Filamin is essential in actin cytoskeletal assembly mediated by p21-activated kinase 1 . Nat Cell Biol 4: 681-690, 2002.

30. Bokoch GM, Wang Y, Bohl BP, Sells MA, Quilliam LA and Knaus UG: Interaction of the Nck adapter protein with p21activated kinase (PAK1). J Biol Chem 271: 25746-25749, 1996. 